\title{
Ciclo de mejora en el aula basado en el aprendizaje en espiral aplicado a los materiales cerámicos.
}

\section{A classroom improvement cycle using the spiral learning on ceramic materials}

Francisco josé García García

ORCID: https://orcid.org/0000-0001-5384-8249

Universidad de Sevilla

Dpto. Ingeniería y Ciencia de los

Materiales y del Transporte

fgarcia49@us.es

DOI: http://dx.doi.org/10.12795/9788447231003.022

Pp.: 466-489 


\section{Descripción del Contexto Académico:}

La asignatura en la que se ha realizado este Ciclo de Mejora en el Aula (CIMA) es Ingeniería de Materiales II, asignatura obligatoria del tercer curso del Grado en Ingeniera Mecánica y de los Dobles Grados en Ingeniería en Diseño Industrial y Desarrollo del Producto e Ingeniería Mecánica y en Ingeniería Eléctrica e Ingeniería Mecánica. La duración del CIMA fue de ocho horas, divididas en cuatro sesiones de dos horas cada una. El tema para el cual se aplicó fue "Materiales Cerámicos". La asignatura cuenta con 167 alumnos matriculados, divididos en dos grupos, entre las tres titulaciones en las que se imparte. Debido a la situación de pandemia actual, donde la Covid-19 nos está machacando, las clases teóricas de la asignatura son $100 \%$ online. Durante la aplicación del CIMA la media de asistencia es de unos 59 alumnos. Por último, hay que destacar que los alumnos demuestran tener conocimientos previos y formas de trabajar en clase muy dispares, como secuencia seguramente de las diferentes titulaciones que provienen. Este hecho es curioso, por cuanto el hecho de estar "protegidos" detrás de las pantallas en casa, junto con el hecho de tener conocimientos y formas de trabajar distintas, provoca que puedan agruparse según patrones de comportamiento bastante distintos: Hay un porcentaje que siempre se mantienen callados, y a los que de verdad cuesta arrancarles respuestas.

Ciclos de Mejora en el Aula (2020). Experiencias de Innovación Docente de la US Esta obra se distribuye con la licencia Creative Commons 


\section{Diseño previo del CIMA}

Mapa de contenidos

La asignatura se divide en cinco bloques, de entre los que destacan los bloques I (aleaciones metálicas) y V (comportamiento en servicio). El CIMA abarca todo el bloque II (materiales cerámicos), si bien se introducirán conceptos del bloque $V$ para que los alumnos se asimilen conceptos como (i) criterios para selección de materiales, (ii) comportamiento en servicio: corrosión, termofluencia, fatiga, desgaste y fractura, (iii) Inspección y ensayos de materiales y (iv) metodología del análisis de fallos de materiales en servicio. Con esto en mente, el mapa de contenidos de este CIMA queda como el que se muestra en la Figura 1.

Como se observa en el mapa de contenidos mostrado en la Figura 1, se realizó un estudio previo de los contenidos a impartir, seleccionándolos por orden cronológico, para que el alumno tome conciencia de como las cerámicas han evolucionado al tiempo que el ser humano ha evolucionado. Los materiales cerámicos y su uso no son frutos puramente del azar. El ser humano ha necesitado tiempo para entender sus propiedades, en disponer de tecnología necesaria para fabricarlos, condicionándose así su desarrollo y explotación, el cómo han dado respuestas en nuevas y novedosas aplicaciones. A mi juicio es importante una metodología en la que el alumno se haga las preguntas que el ser humano viene haciendo desde la antigüedad hasta la actualidad, de manera que comprenda el razonamiento que conduce a la evolución de los mismos.

Ciclos de Mejora en el Aula (2020). Experiencias de Innovación Docente de la US Esta obra se distribuye con la licencia Creative Commons 


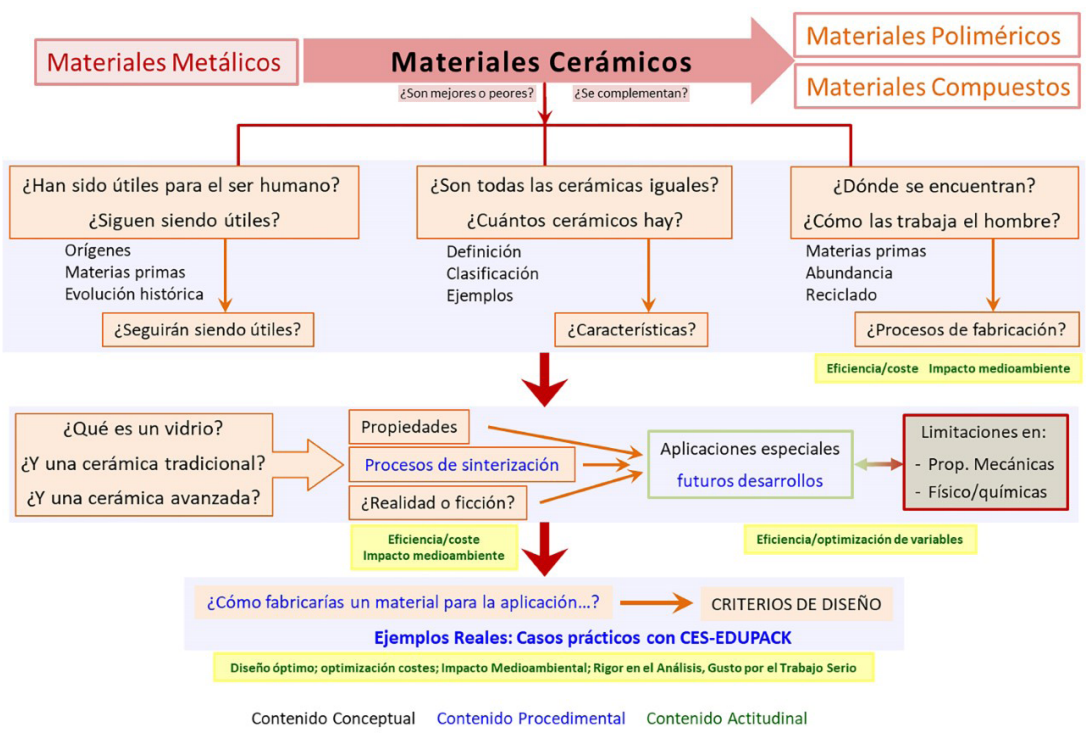

Figura 1. Mapa de contenidos del tema Materiales Cerámicos.jpg

Antes de entrar de lleno con los contenidos propios del tema, se situarán los materiales cerámicos en el conjunto de materiales (metálicos, poliméricos y compuestos), y en especial con los materiales metálicos estudiados en bloque anterior. Esto es algo que se hablará en cada sesión, pues si bien es cierto que un ingeniero mecánico trabaja principalmente con materiales metálicos, también lo es que los materiales cerámicos tienen su sitio, pues tienen unas propiedades que no poseen los materiales metálicos, complementándolos así en una gran variedad de aplicaciones. Es importante hacer ver a los alumnos que cada familia de materiales es única, diferente de las otras, y que son complementarias entre sí, pues todas las familias tienen grandes ventajas e importantes limitaciones. Sólo la solicitación de la pieza en una particular aplicación y el precio del material determinan que familia de material es el más idóneo o menos malo. Por ello es que, ya las preguntas situadas en la parte superior del mapa de contenidos, persigue la diferenciación de los materiales cerámicos con respecto de los demás materiales que forman los distintos bloques de esta asignatura.

Ciclos de Mejora en el Aula (2020). Experiencias de Innovación Docente de la US Esta obra se distribuye con la licencia Creative Commons 
A partir de ahí, se introducirá al alumno en cómo el ser humano ha ido descubriendo y trabajando los diferentes materiales en aplicaciones cada vez más avanzadas, requiriendo piezas más y más complejas, hasta llegar a la actualidad. Para el desarrollo de las cerámicas contamos con la ayuda de un ingeniero-científico que viaja en el tiempo y es un preguntón y pensador nato, y que se va encontrándose con varios problemas a lo largo de la historia. Le surgirán preguntas, tendrá dudas, pero las irá resolviendo con la ayuda inestimable de los alumnos y de la tecnología disponible en esa época.

Posteriormente, se desarrollarán los diferentes tipos principales de materiales cerámicos que existen, desde las cerámicas tradicionales que han ido evolucionando a lo largo del tiempo, a los cerámicos avanzados funcionales o estructurales, enfatizando éstos últimos (son los más relevantes para un ingeniero mecánico). Entraremos de lleno en sus características y propiedades, ventajas y desventajas, avances en las materias primas, métodos de fabricación y en sus aplicaciones.

Finalmente, haremos un ejercicio de ciencia ficción, intentando concebir aplicaciones futuras en función de todo lo aprendido. Para ello, pensaremos en clase posibles aplicaciones de vanguardia en las que estos materiales no tienen rival, y haremos diseños de materiales usando un software ad-hoc para ello. Es importante dejar volar la imaginación, por cuanto esa fantasía es a mi juicio el motor detrás del avance en el desarrollo de los materiales. Creo que un ejercicio de ciencia (o ciencia-ficción) vendrá bien.

El tema, sigue una filosofía de trabajo basada en el "modelo en espiral (Finkel, 2008), y sigue un orden de preguntas que abarcan desde la definición y clasificación de los materiales cerámicos, pasando por cómo lo que son determina sus diferentes propiedades, o como hemos aprendido a fabricarlos y trabajarlos, hasta llegar a los 
criterios de diseño que determinan su uso pasado, presente y futuro. Este método está inspirado en un ejemplo del libro "Lo que hacen los mejores profesores universitarios" (Bain, 2006), basado en el profesor Richard Feynman, quien a partir de ejemplos casi infantiles aumenta gradualmente en dificultad mediante ejemplos de la vida cotidiana. En esta filosofía de trabajo, nuestro ingeniero-científico será un valioso guía y aliado.

A pesar de ser un tema muy teórico, se busca conectar en paralelo contenidos conceptuales (i.e. definición y clasificación) y contenidos procedimentales (i.e. aprender cómo se obtienen y se trabajan...). Así, varias preguntas de nuestro guía van encaminadas en esta dirección. Contenidos actitudinales como ahorro energético, impacto medioambiental de una buena selección de estos materiales, o hacer trabajar rigurosamente para garantizar un óptimo comportamiento en servicio del material seleccionado, también tienen su importancia y se les hará hincapié. Los contenidos actitudinales están implícitos en muchos de los contenidos conceptuales y procedimentales que se tratan en el tema, siendo de extrema importancia en el mundo que vivimos, donde la emisión de gases con efecto invernadero, la contaminación, el crecimiento de la población y la disminución de recursos naturales, entre otros, requiere de una optimización en los materiales permanente y constante de cara a lograr tecnologías cada vez más limpias y eficientes.

\section{Modelo metodológico}

Este modelo metodológico introduce varios cambios respecto de un modelo más tradicional basado en lecciones magistrales. El presente método metodológico, véase la Figura 2, responde bien a mi filosofía de cómo enseñar en clase, introduciendo un problema principal que ocurre en la realidad (una situación real en la vida cotidiana de un ingeniero y que puede tener una o varias soluciones).

Ciclos de Mejora en el Aula (2020). Experiencias de Innovación Docente de la US Esta obra se distribuye con la licencia Creative Commons 
Este método responde siempre a un bucle en el que hay un problema al que hay que darle solución entre todos (los alumnos, nuestro guía y el profesor que guía e instruye por momentos a los estudiantes). Para resolver el problema, nuestro guía se plantea incógnitas y dudas, piensa sobre las herramientas de las que dispone, y se ayuda de los alumnos, formando un sistema de razonamiento crítico en la que crecen intelectualmente tanto los alumnos como nuestro guía... Y de esta forma dar solución o soluciones al problema.

Pero no queda aquí el bucle, pues la resolución del problema dará lugar a otro nuevo problema que por lo general será de mayor complejidad. Así, el razonamiento puesto en práctica con anterioridad sirve como antesala de un nuevo razonamiento donde haya que hacer uso de herramientas nuevas (que pueden ser de tipo conceptual y/o procedimental). Dicho esto, la descripción de cada fase que compone el presente modelo es la siguiente:

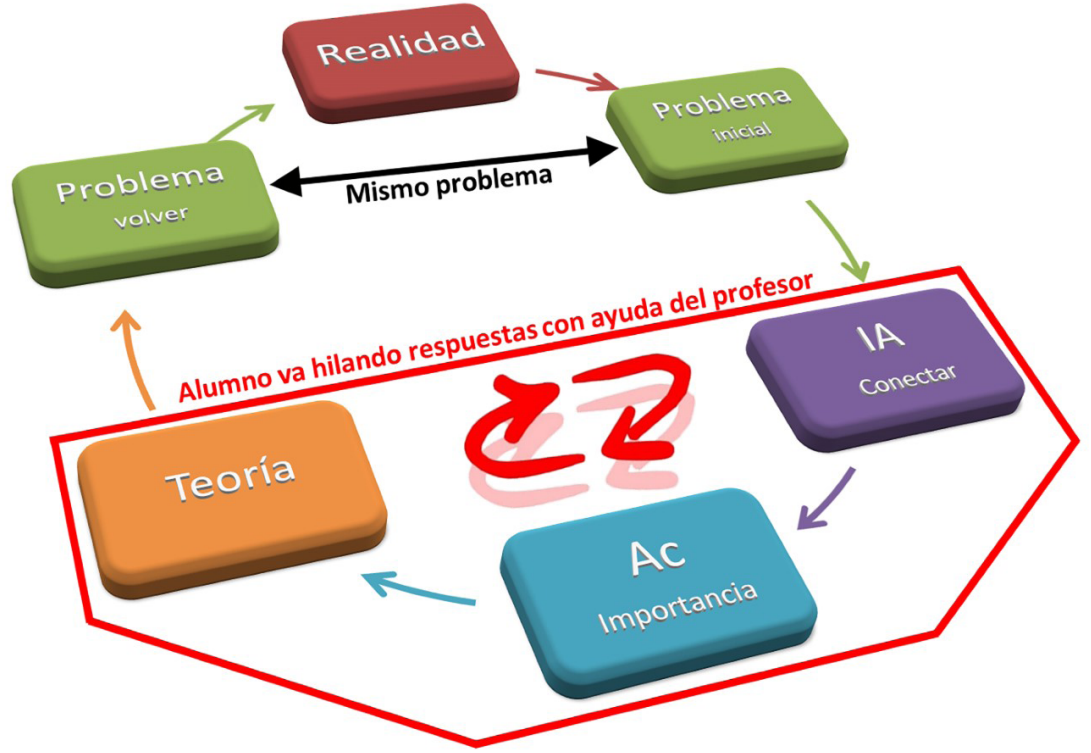

.Figura 2. Modelo metodológico del tema Materiales Cerámicos.

Ciclos de Mejora en el Aula (2020). Experiencias de Innovación Docente de la US Esta obra se distribuye con la licencia Creative Commons 
- Realidad: Esta fase toma este nombre pues antes de plantear el problema en cuestión, ubicamos a nuestro guía en un lugar y tiempo particular, donde observa a su alrededor mejoras posibles en aplicaciones concretas, de cara a mejorar su eficiencia, ser más respetuosa con nuestro medio ambiente, etc. Se introducirá pues el problema en un contexto real.

- Problema inicial: Tras introducir a nuestro guía en un contexto real, se plantea el problema inicial. Este es el punto de partida a la teoría que se pretende enseñar. El problema no es un problema al uso donde se aplican una serie de ecuaciones y se llega a un resultado, sino más bien justo, al contrario. Es decir, nuestro guía tiene inquietudes e imagina que sería ideal realizar una serie de cambios o mejoras, las cuales no sabe bien cómo llevar a cabo, ni tan siquiera si son o no posibles... Bajo esta manera suya de ver y entender las cosas, formulará una pregunta de partida.

Hasta aquí, sólo interviene el guía a través de la voz del profesor. Pero de aquí en adelante, serán los alumnos quienes tengan la batuta de mando y lideren con su participación el desarrollo del modelo metodológico.

Para apoyar nuestro modus operandi, siempre usaremos diapositivas que muestren ejemplos reales de aplicaciones de los distintos tipos de materiales cerámicos que se van a estudiar y el porqué de que haya clasificaciones para ellos, pues estas clasificaciones responden a una serie de características o propiedades que determinan los usos de dichos materiales en aplicaciones diversas.

- Conectar (IA; ideas de los alumnos): Esta fase se llama conectar, por cuanto los alumnos dan rienda suelta a su imaginación, construyendo sus ideas propias. Para ello, es imprescindible conectar conocimientos previos, adquiridos en cursos anteriores o bucles aplicados con

Ciclos de Mejora en el Aula (2020). Experiencias de Innovación Docente de la US Esta obra se distribuye con la licencia Creative Commons 
anterioridad, para de esta forma tomar ideas y pensar sobre ellas (razonamiento crítico). Aquí, profesor y guía callan un tiempo prudencial que variará en función de las preguntas, razonamiento y respuestas que vayan formulando los alumnos. Son ellos quienes piensan a través de sus propias preguntas.

- Importancia (Ac; actividad de contraste): Obviamente, no todos los alumnos progresarán en la fase anterior al mismo ritmo. Algunos pueden no comprender y /o estar de acuerdo con el planteamiento de otros. Puede que el pensamiento crítico se desvíe mucho de los contenidos a enseñar. Para guiar a los alumnos, y tras anotar el desarrollo y contenidos más importantes de su debate en la fase previa, será el propio guía quien reformule con nuevas interrogantes y variables cuan de acuerdo o desacuerdo esté con sus hipótesis/ideas, guiándolos en la dirección de los contenidos que el profesor quiere que adquieran y garantizando que avancen en su manera de razonar y adquiriendo contenidos a la vez, para que no se retrasen unos de otros, o que este retraso sea el mínimo posible. Esta actividad de contraste la denomino importancia, pues persigue que los estudiantes avancen por igual.

- Teoría: Tras estas fases, el profesor entra a formar parte del modelo metodológico por primera vez (hasta ahora hablaba en voz de nuestro guía). El profesor dará nociones teóricas que tendrán un carácter tanto de tipo conceptual como procedimental, para apoyar los razonamientos que llevaron al guía y los alumnos a plantear las posibles ideas de cara a resolver el problema. Aquí, dependiendo de los contenidos a enseñar, se introducirán definiciones, fórmulas, y cuantos contenidos sean necesarios. Pero con una salvedad muy importante con respecto al método tradicional de lecciones magistrales: Los alumnos tomaron conciencia de cómo llegar a estos contenidos y cómo se sitúan en 
un contexto real. De esta forma, pueden ver a través de un razonamiento lógico las respuestas que dieron los grandes pensadores de la época (los porqués).

NOTA: Estas 3 fases (importar, conectar y teoría) están interrelacionadas entre sí, por lo que puede que se repitan más de una vez dentro del bucle principal que responde a este método metodológico. Así, pueden formar un sub-bucle dentro del bucle principal, para evitar introducir todos los contenidos teóricos a la vez, bien porque sean muchos, muy abstractos, de difícil asimilación, o por varias de estas razones. Se busca que los alumnos no pierdan el interés y/o se desconecten por mucho tiempo.

- Problema (volver): Una vez en esta fase, se retoma el problema de partida que tenía nuestro guía, para comprobar los avances realizados por los alumnos y tomar conciencia de cómo a través del pensamiento crítico han ayudado a nuestro guía. Los alumnos han aprendido de los materiales existentes y de sus comportamientos, de las evidencias del entorno, y han conectado así la aplicación real con los conocimientos existentes, para retornar al problema y dar soluciones.

- Realidad: Así, nuestro guía, junto con los alumnos, han ayudado a mejorar lo existente. Pero nuestro guía es muy curioso y está inquieto por mejorar otro aspecto, aplicación, material, etcétera, por lo que, con lo ya aprendido en mente, planteará un nuevo problema que desafie de nuevo a los alumnos.

Y así una y otra vez se repite el modelo metodológico en el CIMA. ¡Es una suerte contar con nuestro amigo ingeniero, curioso y preguntón, para que nos guíe en todos los problemas!

Ciclos de Mejora en el Aula (2020). Experiencias de Innovación Docente de la US Esta obra se distribuye con la licencia Creative Commons 


\section{Secuencia de Actividades}

La secuencia de actividades se recoge en la tabla 1. Las actividades son tanto individuales como en grupos pequeños de unas 5 personas (doy flexibilidad para que se agrupen como quieran y cuantos quieran).

Tabla 1. Secuencia de actividades afín a las cuatro sesiones.

\begin{tabular}{|c|c|c|c|c|c|}
\hline \multicolumn{2}{|c|}{ Actividad } & \multirow{2}{*}{ Descripción } & \multirow{2}{*}{ Medio } & \multirow{2}{*}{ Tiempo } & \multirow{2}{*}{ Contenido } \\
\hline № & Nombre & & & & \\
\hline 1 & Realidad & Se plantean ejemplos reales & \multirow{7}{*}{ E.V. } & \multirow[b]{2}{*}{$15 \min$} & $\mathrm{C}^{*}$ \\
\hline 2 & $\begin{array}{l}\text { Problema } \\
\text { inicial }\end{array}$ & $\begin{array}{l}\text { Se plantea el caso práctico } \\
\text { (cómo diseñar ese material) }\end{array}$ & & & $\mathrm{C} 2^{*}$ \\
\hline 3 & $\begin{array}{l}\text { IA } \\
\text { Conectar }\end{array}$ & $\begin{array}{l}\text { El alumno se cuestiona } \\
\text { los requisitos para elegir } \\
\text { (diseñar) un material y las } \\
\text { posibles alternativas al } \\
\text { mismo }\end{array}$ & & \multirow{3}{*}{$\begin{array}{l}1 \text { hora y } \\
35 \text { min }\end{array}$} & $\mathrm{C} 3^{*}$ \\
\hline 4 & $\begin{array}{l}\text { Ac } \\
\text { Importancia }\end{array}$ & $\begin{array}{l}\text { Optimización de las } \\
\text { propiedades }\end{array}$ & & & $C 4^{*}$ \\
\hline 5 & Teoría & $\begin{array}{l}\text { Asociación de conceptos y } \\
\text { repaso }\end{array}$ & & & $C 5^{*}$ \\
\hline 6 & $\begin{array}{l}\text { Problema } \\
\text { Volver }\end{array}$ & $\begin{array}{l}\text { Asociación de conceptos y } \\
\text { repaso }\end{array}$ & & $8 \mathrm{~min}$ & $C 6^{*}$ \\
\hline 1 & Realidad & $\begin{array}{l}\text { Caso práctico con la que } \\
\text { comenzará la siguiente } \\
\text { sesión }\end{array}$ & & $2 \mathrm{~min}$ & $\mathrm{C} 1^{*}$ \\
\hline
\end{tabular}

C1*: Durante el debate, el contenido consiste en ejemplos reales de materiales cerámicos para diferentes aplicaciones, las cuales irán apareciendo por orden cronológico, de menor a mayor dificultad. Aquí el foco está en debatir porqué uno y no otro material se selecciona para una aplicación concreta, o dicho en otras palabras, que le hace ser el candidato más idóneo. Luego el problema es ¿Cuál es el material más adecuado para la aplicación tal?

C2*: Aquí se plantean casos prácticos que intentan explicar cómo se llegan a las microestructuras y se obtienen

Ciclos de Mejora en el Aula (2020). Experiencias de Innovación Docente de la US Esta obra se distribuye con la licencia Creative Commons 
los microconstituyentes necesarios (como es el material por dentro) para que dicho material sirva o pueda servir en esa aplicación.

- Sesión 1: Vista general a lo largo de la historia.

- Sesión 2: Materiales cerámicos tradicionales y Vidrios.

- Sesión 3: Cerámicas Avanzadas y aplicaciones modernas.

C3*: En este apartado planteamos variantes como diferentes composiciones (como por ejemplo, añadir elementos dopantes) y/o tratamientos térmicos, que dan lugar a materiales con propiedades mecánicas y físico-químicas diferentes. En estas sesiones se hará hincapié en evaluar el efecto que tienen las distintas modificaciones de un material, de cara a alcanzar un compromiso óptimo entre propiedades y aplicación, pues por regla general el mejorar una propiedad suele causar un detrimento en otras propiedades, queremos potenciar.

C4*: Generalmente, suele ocurrir que hay más de una solución para una aplicación en concreto. Es decir, haya varias vías para potenciar alguna propiedad en particular. Sin embargo, otros aspectos de gran relevancia entran ahora en juego, como son el coste de las materias primas y de fabricación, qué método de fabricación es mejor para obtener una pieza de un tamaño y forma concretos, si la aplicación requiere de un material cuya vida útil sea larga o corta (años en servicio previo al fallo), así como del medio exterior (dónde estará esa pieza, si sufrirá cambios bruscos de temperatura, algún tipo de corrosión, esté sometida a esfuerzos mecánicos y/o deformaciones plásticas). Todo ello se revisa en cada sesión, con el objetivo de interrelacionar los conceptos conceptuales y pasados, presentes y futuros.

Ciclos de Mejora en el Aula (2020). Experiencias de Innovación Docente de la US Esta obra se distribuye con la licencia Creative Commons 
El cuestionario propuesto pretende evaluar los mapas mentales de los alumnos antes y después de la aplicación del CIMA. El cuestionario inicial (se procederá igualmente con el cuestionario final) se repartió en Enseñanza Virtual, dando 20 minutos para rellenarlos. Para garantizar el anonimato, se dio la instrucción de que lo firmaran con un alias que recuerden para cuando se les entregue el cuestionario final y que lo enviaran cumplimentados a los delegados de clase, siendo éstos quienes me los enviaron a mí. Así, no tengo forma de saber qué alumno cumplimentó qué cuestionario. El cuestionario inicial se repartió dos sesiones antes de la primera sesión del CIMA, en formato electrónico. Las preguntas, redactadas en lenguaje coloquial y sin que tengan ápice alguno de asimilarse a las de un examen, están diseñadas para ver qué conceptos creen los alumnos que saben aunque en realidad no dominan y viceversa, es decir, que conceptos creen que no saben pero en realidad sí que conocen. Finalmente, antes de comenzar con las preguntas, se indicó en el cuestionario que no se trata de un examen de conocimiento, sino de un ejercicio orientativo para ayudarme a enfocar/orientar la enseñanza del tema. Por tanto, se les pidió verbalmente que lo contestaran con interés.

Las preguntas son las siguientes:

1. ¿Qué piensas que es un material cerámico? Pon dos ejemplos de material cerámico.

2. Di tres propiedades que a tu juicio te parezcan más interesantes para ser material cerámico. Argumenta cada una.

3. ¿Desde cuándo crees que el ser humano trabaja los silicatos?

4. ¿Por qué los materiales cerámicos son frágiles? Argumenta tu respuesta. 
5. Di un requerimiento importante de las cerámicas refractarias. Di un requerimiento importante de las cerámicas abrasivas.

6. ¿Por qué el cemento se endurece al añadir agua?

\title{
Aplicación del CIMA
}

\author{
Desarrollo de las sesiones
}

Sesión 1: La secuencia de actividades ¿Qué aportan los materiales cerámicos respecto del resto de materiales? ¿Cuál ha sido la relación del ser humano con los materiales cerámicos a lo largo de la historia?

NOTA: El bucle se repite 2 veces en esta sesión: Para que los alumnos diferencien entre materiales cerámicos y metálicos. Y para que razonen sobre la evolución de los materiales cerámicos a lo largo de la historia.

Para apoyar nuestro modus operandi o modelo metodológico, partimos de diapositivas que muestran (i) ejemplos reales antiguos de aplicaciones de materiales metálicos (espada, escudo) y cerámicos (utensilios de cocina). (ii) Evolución de los materiales cerámicos en el tiempo (desde vasijas prehistóricas, hasta porcelana China, introducida en Europa por Maco Polo, y que no se reprodujo en Europa hasta la edad moderna). Estas diapositivas pretenden concienciarles del porqué que haya clasificaciones para ellos, y cómo estas clasificaciones responden a una serie de características o propiedades que determinan los usos de dichos materiales. Además, se les ilustra con imágenes de sus microestructuras y sus arreglos atómicos, los cuales ellos deben identificar y asociar.

Ciclos de Mejora en el Aula (2020). Experiencias de Innovación Docente de la US Esta obra se distribuye con la licencia Creative Commons 
Los alumnos dispusieron de varios minutos para discutir (identificar y asociar) por ellos mismos a qué cerámica pertenece cada microestructura y arreglo atómico. Además, nuestro guía les preguntó (añadiendo variables) del porqué esa estructura determina qué propiedades, de manera que se aplique de una manera u otra. De esta forma, los alumnos tuvieron que pensar cómo los diferentes tipos de enlaces, tamaños de grano, procesos de sinterización, etcétera, nos permiten anticipar un determinado comportamiento en servicio. Esta sesión es de tipo introductoria por cuanto persigue que el alumno tome conciencia de las materias primas que se emplean en la fabricación de las cerámicas, de sus orígenes y sus aplicaciones, de cómo se diferencian y complementan a los materiales metálicos. De cómo ha sido desarrollo o evolución.

Tras este periodo largo de reflexión, el profesor explicó la teoría general (afín a todos los materiales cerámicos) de los enlaces cristalinos y las características de los mismos. De esta forma, se interrelacionó teoría, conceptos surgidos de sus ideas y actividad de contraste. Finalmente, se introdujo la pregunta ¿Y cómo clasificarías tú los materiales cerámicos? ¿Por qué? Que dará lugar a las sesiones 2 y 3.

Sesión 2: ¿Cómo clasificarías los materiales cerámicos? ¿Qué materiales (y por qué) aplicarías en un vaso de precipitado de laboratorio, y para una vitro-cerámica, y para construcción de edificios, y para lijar un metal oxidado?

En esta sesión, y antes de mostrar diapositivas, los alumnos razonaron críticamente por ellos mismos y dieron respuestas con sus ideas. Lógicamente, éste es nuestro punto de partida. Aquí se generó un gran debate que despertó el interés de la mayoría de los alumnos, siendo partícipes de la importancia de lo que se va a estudiar. Nuestro guía introdujo mediante actividades de contraste ciertas

Ciclos de Mejora en el Aula (2020). Experiencias de Innovación Docente de la US Esta obra se distribuye con la licencia Creative Commons 
ideas que ayuden a encaminarles en la dirección correcta tras varios minutos de debate, a la vez que apuntó las conclusiones. Nuestro ingeniero guía da pistas para identificar las microestructuras y arreglos y repetimos el juego $(I A+A C)$. Los alumnos definen propiedades físico-químicas e ingenieriles (mecánicas), si bien es posible que no todas, puede que algunas tengan claro a qué características del material se deben. Tras este periodo de conexión, el profesor identificó los materiales y sus características que determinan las propiedades más significativas y que les confieren un uso u otro (teoría). Para ello se valió de las diapositivas previamente preparadas. Y lanza a los estudiantes la pregunta: ¿Y cómo se llega a una estructura u otra? ¿Qué consecuencias tiene? ¿Y qué pasa si...? Estas preguntas se mantuvieron a lo largo de todo el problema planteado en esta sesión 2. Se pretende así lanzar el gancho para hacer partícipes a los estudiantes, a la vez que les anime a tener inquietudes propias y auto-responderse ¿Y por qué? ¿Y por qué? ¿Y por qué? ¿Y por qué?

Una vez tenemos todas las herramientas a nuestra disposición, retornamos al problema de origen para ayudar a nuestro guía a resolverlo. Como es lógico cuando se plantea una interrogante detrás de otra, se espera que los alumnos planteen y resuelvan otros problemas menores que surjan en el camino, como respuestas a esos "¿y por qué?". La sesión 2 finalizó con la pregunta ¿Qué se te ocurre para mejorar las propiedades de los materiales cerámicos?

Sesión 3: ¿Cómo se pueden mejorar las prestaciones de los materiales cerámicos estudiados? ¿Qué aplicaciones nuevas se te ocurren?

En esta sesión, muy similar a la anterior, los alumnos razonaron críticamente por ellos mismos y aportaron sus ideas sobre los que piensan para dar respuestas (IA). De 
nuevo, surgió un gran debate, por cuanto aquí la imaginación jugará un papel muy importante (maneras que ni se me ocurren a mí ahora mismo sobre cómo mejorar los cerámicos, aplicaciones quizá de los más extravagantes...). De nuevo, nuestro guía aportó ideas de cómo mejorar y dónde aplicar o no aplicar los materiales cerámicos mejorados (AC), introdujo mediante actividades de contraste ideas que ayuden a encaminarles en la dirección correcta. Hay que notar que en esta sesión, los alumnos dispusieron de mayor tiempo para aportar sus ideas, tomando así más liderazgo/participación en esta sesión que en la anterior. Como siempre, se apuntaron las conclusiones. Tras el debate, nuestro guía dio pistas para identificar las microestructuras y arreglos, y repetimos el juego $(I A+A C)$. Tras este periodo de IA + Ac, el profesor desarrolló los parámetros más significativos que dan lugar a la evolución y desarrollo de las cerámicas modernas o avanzadas, y que se clasifican en estructurales y funcionales. Por supuesto, se hizo hincapié en las propiedades físico-químicas e ingenieriles de las mismas, a la vez que se dieron ejemplos de estas cerámicas en la vida real. Esta sesión reforzará sus conocimientos sobre el impacto que tiene la variación de ciertos parámetros (incluido el uso de una materia prima u otra) en las propiedades finales, lo que les permitirá anticipar cómo optimizar las piezas para una aplicación u otra.

Al finalizar la sesión, los alumnos cuentan con un razonamiento crítico y unos conocimientos conceptuales, procedimentales y actitudinales suficientes para poder anticipar qué material o materiales cerámicos usar en aplicaciones específicas. Por ello, al final de la sesión, se lanzó la pregunta: Si diseñaras una pieza para una aplicación cualquiera, ¿qué material o materiales usarías y por qué?

Ciclos de Mejora en el Aula (2020). Experiencias de Innovación Docente de la US Esta obra se distribuye con la licencia Creative Commons 
Sesión 4: ¿Qué material usarías para la aplicación que estés pensando ahora?

En esta sesión, y tras un debate entre alumnos y nuestro guía, se escogieron varias aplicaciones donde ellos pondrán de manifiesto su razonamiento crítico todo lo aprendido, para diseñar y optimizar los materiales a emplear (así como su coste y procesos de fabricación) en piezas que sirvan para estas aplicaciones. Por tanto, el fin de esta última sesión es el diseño de materiales cerámicos en función de las aplicaciones a las que llegamos en consenso. A priori, esta actividad estaba pensada para que la hicieran en grupos, de manera que se repartieran las aplicaciones que pensáramos entre todos, incluido nuestro curioso guía. Y digo esto porque tenía pensado llevarlos a una de las aulas informáticas de la Escuela Politécnica Superior (centro donde se debería impartir la asignatura de no ser por la Covid-19). Estas aulas disponen de un programa llamado CES-EDUPACK que permite seleccionar materiales en función de parámetros y condiciones de contorno seleccionados (corrosión a un medio o varios medios, densidad, temperatura de aplicación, resistencia máxima a impacto, vida útil, etc). Sin embargo, a causa de la Covid-19, se hizo usando mi ordenador conectado en remoto al centro de cálculo de la Escuela Politécnica Superior. Aun así, creo que fue muy divertida, por cuanto los alumnos pusieron en práctica lo aprendido en ejemplos reales. Ni que decir tiene que nuestro guía propuso dos aplicaciones "trampa", por cuanto necesitan de más de un material (sea el caso de un vidrio electrocrómico o ventana inteligente), desafiándoles así a pensar en combinaciones de materiales para estas aplicaciones, bien sea varios cerámicos, o bien cerámico + no cerámico. Y todo ello lo aproveché para introducir el concepto de Ingeniería Forense (bloque $\mathrm{V}$ de la asignatura), que se entiende bien con el siguiente ejemplo: Tenemos que ser conscientes que las cosas terminan fallando, por suerte, en la mayoría de los casos por un Ciclos de Mejora en el Aula (2020). Experiencias de Innovación Docente de la US 
cúmulo de circunstancias asociadas a las complejas condiciones de servicio, que hacen muy complicado definir la causa del fallo y por tanto, establecer un protocolo de mantenimiento o actuación para que no vuelva a ocurrir. Por ejemplo, si a un paciente le da un infarto, la causa es clara, murió de un fallo en el corazón. Si buscamos una analogía evidente en Ingeniería, sin un avión no tienen sus alas, NO podrá volar. Otro caso, muy diferente es saber la causa de la muerte de un paciente que tienen varias enfermedades relacionadas entre sí. Cuando en ingeniería, una pieza o un componente está sometido a condiciones complejas y extremas (mecánicas, desgaste, corrosión, temperatura), cuando falle, será bastante complicado asegurar 100 \% por qué falló, ahí está el papel y el grado de experiencia de los ingenieros e investigadores que se dedican a esta rama (Ingeniería Forense), que es lo que trabajamos en esta práctica.

Con esta metodología aplicada en las 8 horas que constituyen el CIMA, se pretende que los estudiantes guíen la clase, comprendiendo por ellos mismos cómo se llega a la obtención de dichas microestructuras y arreglos atómicos, y como éstos están ligados a las diferentes propiedades que hacen que destaquen en aplicaciones concretas. Por otro lado, el que los alumnos vayan a la delantera de la resolución del problema me sirve conocer sus defectos para reforzarlos, sobre todo aquellos relacionados con una falta de nivel de base de cursos anteriores. También que ellos vean como esta interrelación de conceptos se les pondrá de manifiesto el día que salgan de la universidad y comiencen la vida laboral. Básicamente se trata de que vean cómo la teoría apoya a la práctica que están viendo a través de un ejemplo real y atractivo para ellos. En el mundo de los materiales y sus comportamientos en servicio, no hay una única solución para cada aplicación. Hay muchas variables y es muy importante que entiendan los porqués que hay detrás. Es lo que he denominado como IA (conectar) + Ac (importancia) + Teoría.

Ciclos de Mejora en el Aula (2020). Experiencias de Innovación Docente de la US Esta obra se distribuye con la licencia Creative Commons 
La evaluación de los modelos mentales y del aprendizaje de los alumnos se ha realizado mediante la comparación de las respuestas del cuestionario inicial y final, que se pasaron pasado antes y después de la aplicación del CIMA (Porlán, 2017). Este cuestionario fue realizado por 59 alumnos. Se analizaron 37 de ellos, puesto varios alumnos respondieron el cuestionario final con un alias distinto al inicial. Las respuestas a cada una de las seis preguntas se clasifican en tres escalones en función del nivel complejidad de la respuesta, siendo de nivel más básico a más avanzado: $\mathrm{A}=$ Respuesta incorrecta o en blanco; $\mathrm{B}=$ Definición correcta, sin justificar (razonar), ni precisar detalles o incluir ejemplos; $\mathrm{C}=$ Respuesta correcta, razonada y bien justificada, interrelacionando con otros conceptos. El análisis de evaluación del aprendizaje basado en los cuestionarios inicial y final CIMA se recoge en las Figura 3.

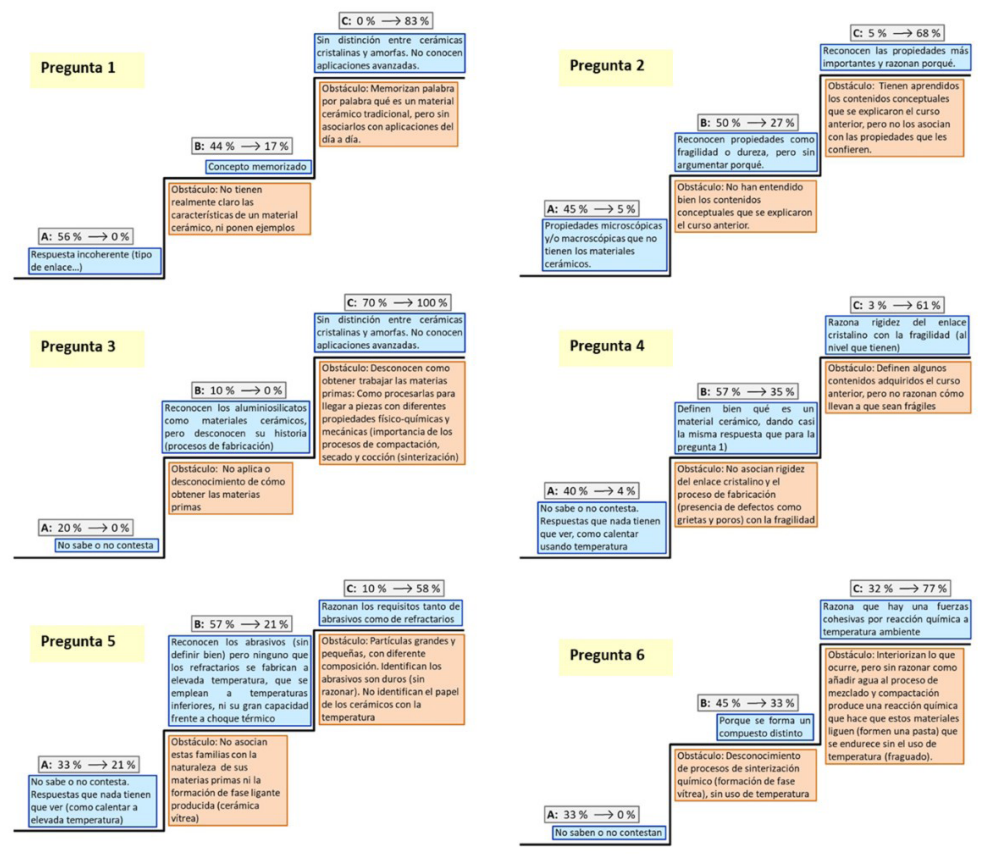

Figura 3. Escaleras de aprendizaje de los alumnos.

Ciclos de Mejora en el Aula (2020). Experiencias de Innovación Docente de la US Esta obra se distribuye con la licencia Creative Commons 
Las dos conclusiones principales que se pueden extraer de la evaluación tras el análisis de las respuestas son: (i) Que el CIMA ha ayudado a que los modelos mentales de la mayoría de los alumnos pasen de desconocimiento a nivel medios-avanzados. De hecho, la mayoría de ellos ha pasado del escalón A al escalón C en casi todas las respuestas. (ii) Y que los alumnos razonan de manera crítica que son los diferentes tipos de materiales cerámicos, desde los más tradicionales a los más avanzados, sus características y propiedades, los métodos de fabricación y, quizá los más importante, entrelazar todos ellos para elucidar sus aplicaciones.

Una conclusión menor es que también han interiorizado contenidos conceptuales previos, los cuales estaban memorizados en sus modelos mentales, pero que no lograban comprender. En este sentido, el cuestionario inicial ha sido especialmente importante, por cuanto me permitió sacar la conclusión principal que los modelos mentales de muchos alumnos no estaban a la altura de lo que yo como docente esperaba de ellos. Los contenidos conceptuales y los nexos de unión entre los mismos no estaban lo suficientemente interiorizados en muchos estudiantes, lo que me obligó a replantearme el nivel de partida y como estimularlos en clase.

Sin embargo, como crítica el procedimiento empleado, hay que señalar que los cuestionarios lo cumplimentaron en casa a consecuencia de la docencia telemática establecida a causa de la pandemia. Por ello, es difícil asegurar al $100 \%$ que los alumnos respondieron al cuestionario sin buscar la información en la red mediante el uso de dispositivos electrónicos. Este posible falseamiento de los datos de análisis puede llevar al docente a establecer hipótesis y evaluaciones de los alumnos erróneas. La verdad es que me hubiese gustado pasar el cuestionario en clase, de forma que se pudiera evitar la duda en las respuestas. 


\section{Evaluación del CIMA}

\section{Cuestiones a mantener y cambios a introducir para un futuro CIMA}

En líneas generales estoy satisfecho con el resultado obtenido tras la aplicación del CIMA. La unión del aprendizaje en espiral y el método del taller conceptual, donde los alumnos trabajan sobre problemas diseñados alrededor de un enigma, les permitió adquirir un razonamiento crítico con el que resolver problemas ahora y en el futuro, al mismo tiempo que ganar en conocimiento. Además, centrar la enseñanza en ellos me ha permitido verles participar en clase (a una parte de ellos) como no veía desde hace tiempo. Sentirme partícipe de su evolución mediante razonamiento crítico, de cómo los alumnos relacionan contenidos conceptuales y procedimentales con aspectos de la vida cotidiana que no se habían detenido a pensar, pero que tienen asimilados sin saberlo, me causó una grata sensación.

Dos aspectos importantes a mantener a partir de ahora son (i) el uso de los cuestionarios y su evaluación, pues me ayudaron mucho para anticiparme a los modelos mentales de los alumnos y sus obstáculos principales. (ii) El diario de sesiones o diario del profesor ha sido otra herramienta que me ha impresionado gratamente, pues me ha permitido desarrollar mi trabajo de autoevaluación y de evaluación del diseño didáctico aplicado. Estas herramientas eran desconocidas y son con diferencia las que más me han ayudado.

Respecto de los cambios a introducir en futuros ciclos de mejora, sin duda cambiaría algunas de las preguntas utilizadas en los cuestionarios, principalmente por la manera de formularlas, que no eran complemente apropiadas 
al ser muy genéricas. Esto me dificultó la clasificación de las respuestas por niveles. También introduciría cambios con actividades adicionales, como vídeos que sirvieran de material suplementario y la resolución de ejercicios de elevada dificultad. Es cierto que se les ha proporcionado a los alumnos las herramientas necesarias para resolver los ejercicios, a la vez que un razonamiento crítico que antes no tenían. Sin embargo, aplicar esta metodología durante los ciclos de mejora me ha retrasado mucho respecto del otro grupo, lo que me obliga a reducir contenidos de una u otra forma. Esta ha sido mi dificultad mayor, y para la cual no tengo respuesta hoy día. Por ello creo que algo que cambiaría sería los contenidos, bien por su cantidad o bien reestructurándolos de forma distinta. La pregunta ahora es ¿cómo?

\section{Principios Didácticos que han guiado la experiencia}

Los principios didácticos que me han guiado son el uso del mapa de contenido y modelo metodológico diseñados a conciencia que discriminen lo realmente importante y necesario, junto con el uso de preguntas para atraer al alumno. También el uso de cuestionarios bien diseñados para conocer los modelos mentales de cada uno de los estudiantes y tener una visión cercana a la realidad del alumno. Y el diario del profesor para anotar cuanto acontece y tenerlo presente en un futuro. Por último, una reflexión sobre el mapa de contenidos, modelo metodológico y cuestionario empleados, que han demostrado tener varios puntos débiles que quiero modificar para mejorarlos en un futuro.

Ciclos de Mejora en el Aula (2020). Experiencias de Innovación Docente de la US Esta obra se distribuye con la licencia Creative Commons 
Palabras claves: Materiales Cerámicos, Ingeniera de Materiales II, Docencia Universitaria, Experimentación Docente Universitaria, Ingeniería Mecánica, Doble Grado en Eléctrica y Mecánica, Doble Grado en Diseño Industrial y Desarrollo del Producto e Ingeniería Mecánica.

Keywords: Ceramic materials, Materials Engineering II, University teaching, University teaching experimentation, Mechanical engineering, Double Bachelor's degree in Electrical and Mechanical Engineering, Double Bachelor's degree in Industrial design and Product development, and Mechanical Engineering.

\section{Referencias}

Bain, K. (2005). Lo que hacen lo mejores profesores universitarios. Valencia: Universidad de Valencia.

Finkel, D. (2008). Dar clase con la boca cerrada. Valencia: Universidad de Valencia.

Porlán, R. (coord.) (2017). Enseñanza Universitaria. Cómo mejorarla. Madrid: Editorial Morata.

Ciclos de Mejora en el Aula (2020). Experiencias de Innovación Docente de la US Esta obra se distribuye con la licencia Creative Commons 